\title{
Comparison of Decidual Vasculopathy in Central and Peripheral Regions of Placenta with Implication of Lateral Growth and Spiral Artery Remodeling
}

\author{
Peilin Zhang \\ Pathology, New York Presbyterian-Brooklyn Methodist Hospital, Brooklyn, NY 11215, USA; Pez9008@nyp.org
}

Received: 10 September 2020; Accepted: 29 September 2020; Published: 2 October 2020

\begin{abstract}
Decidual vasculopathy at late gestation was shown to be associated with spiral artery remodeling at implantation. Dramatic decidual vascular transformation from early to late stage pregnancy suggests a dynamic spatiotemporal relationship between the various vascular components in spiral artery remodeling and decidual vasculopathy. The central and peripheral portions of 105 placentas with decidual vasculopathy at term were examined with or without preeclampsia to see if temporal vascular regeneration was present. Central and peripheral vasculopathy and central and peripheral regeneration were compared. The peripheral portion showed more decidual vasculopathy (88 of total 105, 83.8\%) than central portion (72 of total 105, 68.6\%, $p<0.0001$ ). However, central portion showed more vascular regeneration (51 of total 105,48.6\%) than the peripheral portion (23 of total 105, 21.9\%, $p<0.0001$ ). There was no difference in vasculopathy or regeneration with or without preeclampsia. Spiral artery remodeling is non-synchronous during placental growth and vascular regeneration. This spatiotemporal sequence may help interpretation of morphologic changes of decidual vasculopathy.
\end{abstract}

Keywords: decidual vasculopathy; spiral artery remodeling; placenta lateral growth; maternal vascular regeneration

\section{Introduction}

Placental growth is continuous throughout the pregnancy to reach maturity at term [1,2]. The size and weight of the placenta are independent factors affecting the fetal growth and fetal wellbeing [3-7]. Placenta in a large part demonstrates a lateral growth pattern due in part to the mechanical limit of the uterine cavity with continuous expansion of a number of cotyledons from the periphery [1]. Like many other mammalian organs, there are regulatory mechanisms of size control and cell number control in the growth of placenta [8-10]. Development of cotyledons laterally from the center involves the development of new anchoring villi, trophoblastic cell shell and column with corresponding spiral artery remodeling so that the fetal villous vessels are connected to the uteroplacental intervillous circulation [1]. It is reasonable to believe that there is a time sequence (temporal sequence) in regard to spiral artery remodeling from the center where spiral artery remodeling occurs first and peripheral part of the placenta later (spatial sequence), although the spatiotemporal sequence of placental development is not frequently studied [11,12]. Spiral artery remodeling is a significant vascular transformation of maternal vessels to accommodate the embryonic and placental growth and to establish the utero-placental circulation [13]. Spiral artery remodeling is a temporal but dynamic development process predominantly occurring in the first and second trimester [1]. Based on previous studies, there is no morphologic evidence of spiral artery remodeling after the end of the second trimester [14]. The size of the placenta increases to about $20 \mathrm{~cm}$ in diameter and 25-40 cotyledons at term from a blastocyst at the beginning of implantation, although the most significant placenta 
weight increase occurs in the third trimester [1]. The questions remain if the spiral artery in the central portion of the placenta and those in the peripheral portion show synchronous morphologic changes of remodeling, and how this difference of spiral artery remodeling in the time between the central and peripheral portions of placenta affects the development of decidual vasculopathy at late gestation, and how this spatiotemporal spiral artery remodeling affects the interpretation of morphologic changes of placenta in normal pregnancy and complications. The spatiotemporal difference is typically not an issue for most placental pathologists when the placental surface is examined and decidual vasculopathy found, as the entire placenta can be easily accessed after delivery. However, the placental bed biopsy interpretation of maternal vessels will be affected significantly due to the tissue sections obtained from the biopsy sites at the placental bed [15]. The central portion of the placental bed is emphasized for placental bed biopsy in order to achieve the expected results [15]. Under these circumstances, the spatiotemporal sequence of maternal vascular changes makes a difference in interpretation of the material obtained for the procedure [15]. Furthermore, the theory of "failure to invade" has been proposed as the key pathogenic mechanism of preeclampsia and hypertensive disorders of pregnancy, and the cardinal vascular change of "failure to invade" theory is the lack of trophoblastic invasion of vascular wall in the superficial myometrium. In this setting, an attempt was made to delineate the difference of morphologic features of the maternal vessels within the center and the peripheral areas of maternal surface in regards to the presence and absence of decidual vasculopathy and vascular regeneration/restoration. A slight modification of standard placental examination protocol for a period of this study was made in order to answer this question.

\section{Material and Methods}

The study is exempt from Institutional Review Board (IRB) approval according to section 46.101(b) of 45CFR 46 which states that research involving the study of existing pathological and diagnostic specimens in such a manner that subjects cannot be identified is exempt from the Department of Health and Human Services Protection of Human Research Subjects. Placentas submitted for pathology examination for a variety of clinical indications were included in the study and examined under the standard protocol with the following modification of gross dissection and examination. Standard four sections were submitted for microscopic examination with one maternal surface section at the center and one at the periphery using the horizontal dissection method (en face) previously described (Figure 1) [16]. A total of four sections per placenta were included with one full thickness section from the peripheral area excluding the margins and one section of umbilical cord and membrane roll (Figure 1). Routine examination of placenta was performed under the clinical practice guideline using the Amsterdam criteria [17]. Diagnostic criteria for decidual vasculopathy was according to the guideline previously described $[17,18]$. Vascular regeneration/restoration (recovery) of maternal spiral artery was defined by the presence of endothelium and smooth muscle within the vascular lumen as well as intramural or endovascular trophoblasts. The presence of intramural and/or endovascular trophoblasts (acute atherosis) and fibrinoid medial necrosis of vascular wall were interpreted as the presence of decidual vasculopathy $[1,18,19]$. Immunohistochemical staining procedures were performed on paraffin-embedded tissues using Leica Biosystems Bond III automated immunostaining system following the manufacturer's instructions. Monoclonal antibodies against CD56, WT1, and smooth muscle myosin heavy chain (SMYOHC) were purchased from Agilent (Dako Corporation, Santa Clara, CA, USA) for clinical in vitro diagnostics with appropriate dilutions (CD56 1:100; WT1 1:400; SMYOHC 1:400) and controls under catalogue numbers M730401-2, M3561, and M7165. Selective cases of decidual vasculopathy were confirmed by the presence of CD56 for endovascular or intramural trophoblasts, and the regeneration by presence of WT1 and smooth muscle myosin heavy chain (SMYOHC), as WT1 gene expression is known to be present in endothelial cells and smooth muscle cells of vessels [20,21]. Immunostaining for these markers was only used in selected cases to illustrate the usefulness of the techniques, and the data was based on the morphologic features. 


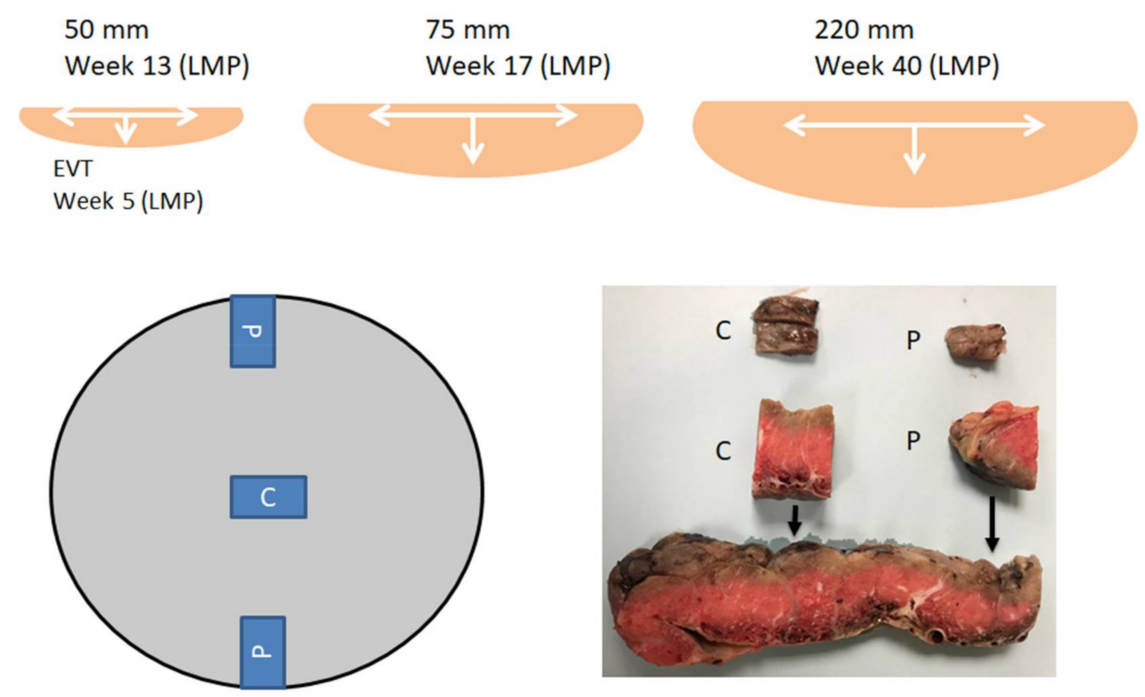

Figure 1. Schematic representation of placental lateral growth from early pregnancy to term with representative dissection techniques of Khong (en face sectioning of decidua) for placental surface and decidual vessels. C, central region; P, peripheral region of maternal surface; EVT, Endovascular trophoblasts; LMP, last menstrual period.

\section{Result}

The diagram (Figure 1) showed the theoretical placental lateral growth with expansion from the center to the periphery. Endovascular trophoblasts (EVT) can be identified within the spiral artery at week five from the last menstrual period (LMP). The bottom panel showed the method of gross dissection of placental surface at the center and the periphery using the method of Khong for better sampling and visualization of decidual vessels (en face decidual section) [16]. Using this method of gross sectioning, we examined 105 placentas including 14 placentas from preeclampsia and 91 placentas from other complications without preeclampsia. The baseline characteristics of all the placentas with related clinical and pathologic findings are shown in Table 1. The baseline characteristic data from the current study was similar to those reported previously [18].

Table 1. Characteristics of 105 placentas with decidual vasculopathy.

\begin{tabular}{cccc}
\hline Groups & Classic Type $(n=87)$ & Mixed Type $(n=18)$ & $p$-Value \\
\hline Delivery & & & 0.492 \\
-C-section & $33(37.9 \%)$ & $9(50.0 \%)$ & \\
- Vaginal & $54(62.1 \%)$ & $9(50.0 \%)$ & \\
PIH & & & 0.002 \\
0 & $80(92.0 \%)$ & $11(61.1 \%)$ & \\
1 & $7(8.0 \%)$ & $7(38.9 \%)$ & \\
Weight & $456.8 \pm 122.0$ & $345.3 \pm 106.6$ & 0.000 \\
Gestational age $(\mathrm{W})$ & $40.0[38.5 ; 40.0]$ & $39.0[36.0 ; 40.0]$ & 0.025 \\
Infarcts & & & 0.585 \\
0 & $75(86.2 \%)$ & $14(77.8 \%)$ & \\
1 & $12(13.8 \%)$ & $4(22.2 \%)$ & 0.381 \\
Chorioamnionitis & & & \\
0 & $31(35.6 \%)$ & $9(50.0 \%)$ & 0.584 \\
1 & $56(64.4 \%)$ & $9(50.0 \%)$ & \\
Meconium & & & \\
0 & $49(56.3 \%)$ & $6(33.3 \%)$ & \\
1 & $38(43.7 \%)$ & &
\end{tabular}


Table 1. Cont.

\begin{tabular}{|c|c|c|c|}
\hline Groups & Classic Type $(n=87)$ & Mixed Type $(n=18)$ & $p$-Value \\
\hline Thrombosis & & & 1.000 \\
\hline 0 & $65(74.7 \%)$ & $13(72.2 \%)$ & \\
\hline 1 & $22(25.3 \%)$ & $5(27.8 \%)$ & \\
\hline GDM2 & & & 0.603 \\
\hline 0 & $79(90.8 \%)$ & $15(83.3 \%)$ & \\
\hline 1 & $8(9.2 \%)$ & $3(16.7 \%)$ & \\
\hline Category 2 tracing & & & 0.037 \\
\hline 0 & $65(74.7 \%)$ & $18(100.0 \%)$ & \\
\hline 1 & $22(25.3 \%)$ & $0(0.0 \%)$ & \\
\hline Villitis & & & 0.371 \\
\hline 0 & $72(82.8 \%)$ & $17(94.4 \%)$ & \\
\hline 1 & $15(17.2 \%)$ & $1(5.6 \%)$ & \\
\hline Abruption & & & 0.126 \\
\hline 0 & $86(98.9 \%)$ & $16(88.9 \%)$ & \\
\hline 1 & $1(1.1 \%)$ & $2(11.1 \%)$ & \\
\hline IUGR & & & 0.038 \\
\hline 0 & $83(95.4 \%)$ & $14(77.8 \%)$ & \\
\hline 1 & $4(4.6 \%)$ & $4(22.2 \%)$ & \\
\hline Cord issues & & & 0.755 \\
\hline 0 & $82(94.3 \%)$ & $16(88.9 \%)$ & \\
\hline 1 & $5(5.7 \%)$ & $2(11.1 \%)$ & \\
\hline Others & & & 1.000 \\
\hline 0 & $80(92.0 \%)$ & $16(88.9 \%)$ & \\
\hline 1 & $7(8.0 \%)$ & $2(11.1 \%)$ & \\
\hline Cord coiling & $3.0[1.0 ; 4.0]$ & $3.5[2.0 ; 5.0]$ & 0.225 \\
\hline Oligohydramnios & & & 1.000 \\
\hline 0 & $85(97.7 \%)$ & $17(94.4 \%)$ & \\
\hline 1 & $2(2.3 \%)$ & $1(5.6 \%)$ & \\
\hline Central & & & 0.930 \\
\hline 0 & $28(32.2 \%)$ & $5(27.8 \%)$ & \\
\hline 1 & $59(67.8 \%)$ & $13(72.2 \%)$ & \\
\hline Peripheral & & & 1.000 \\
\hline 0 & $14(16.1 \%)$ & $3(16.7 \%)$ & \\
\hline 1 & $73(83.9 \%)$ & $15(83.3 \%)$ & \\
\hline Both C + P (DV) & & & 0.64 \\
\hline 0 & $42(48.3 \%)$ & $7(38.9 \%)$ & \\
\hline 1 & $45(51.7 \%)$ & $11(61.1 \%)$ & \\
\hline Recovery (Central) & & & 0.695 \\
\hline 0 & $46(52.9 \%)$ & $8(44.4 \%)$ & \\
\hline 1 & $41(47.1 \%)$ & $10(55.6 \%)$ & \\
\hline Recovery (Peripheral) & & & 1.000 \\
\hline 0 & $68(78.2 \%)$ & $14(77.8 \%)$ & \\
\hline 1 & $19(21.8 \%)$ & $4(22.2 \%)$ & \\
\hline Both $(C+P)$ PRR & & & 1.000 \\
\hline 0 & $76(87.4 \%)$ & $16(88.9 \%)$ & \\
\hline 1 & $11(12.6 \%)$ & $2(11.1 \%)$ & \\
\hline Both $(C+P)$ ARR & & & 0.982 \\
\hline 0 & $50(57.5 \%)$ & $11(61.1 \%)$ & \\
\hline 1 & $37(42.5 \%$ & $7(38.9 \%)$ & \\
\hline
\end{tabular}

Table 1: Baseline characteristics of 105 placentas with decidual vasculopathy and other clinical and pathologic findings within the placentas. $0=$ absence of the change, $1=$ presence of the change. Cord issues include abnormal insertions, knots, length, and cord vessels. Others include fetal anomalies, fetal vascular malperfusions, chorangiosis/chorangiomas, maternal gestational cholestasis, maternal autoimmune diseases, etc. Cord coiling refers to numbers of coils per $10 \mathrm{~cm}$ of cord. Both $\mathrm{C}+\mathrm{P}(\mathrm{DV})$ indicates the presence of decidual vasculopathy at both the central and peripheral regions. Both $(C+P) P R R$ and Both $(C+P)$ ARR depict the presence and the absence of vascular regeneration/restoration in both the central and the peripheral regions. 
The presence of decidual vasculopathy (DV) at the center and the periphery of placentas can be easily identified under light microscope (Figure 2). The morphologic features of decidual vasculopathy are those described previously. Vascular regeneration after transformation can also be readily identified under light microscope (Figure 2).

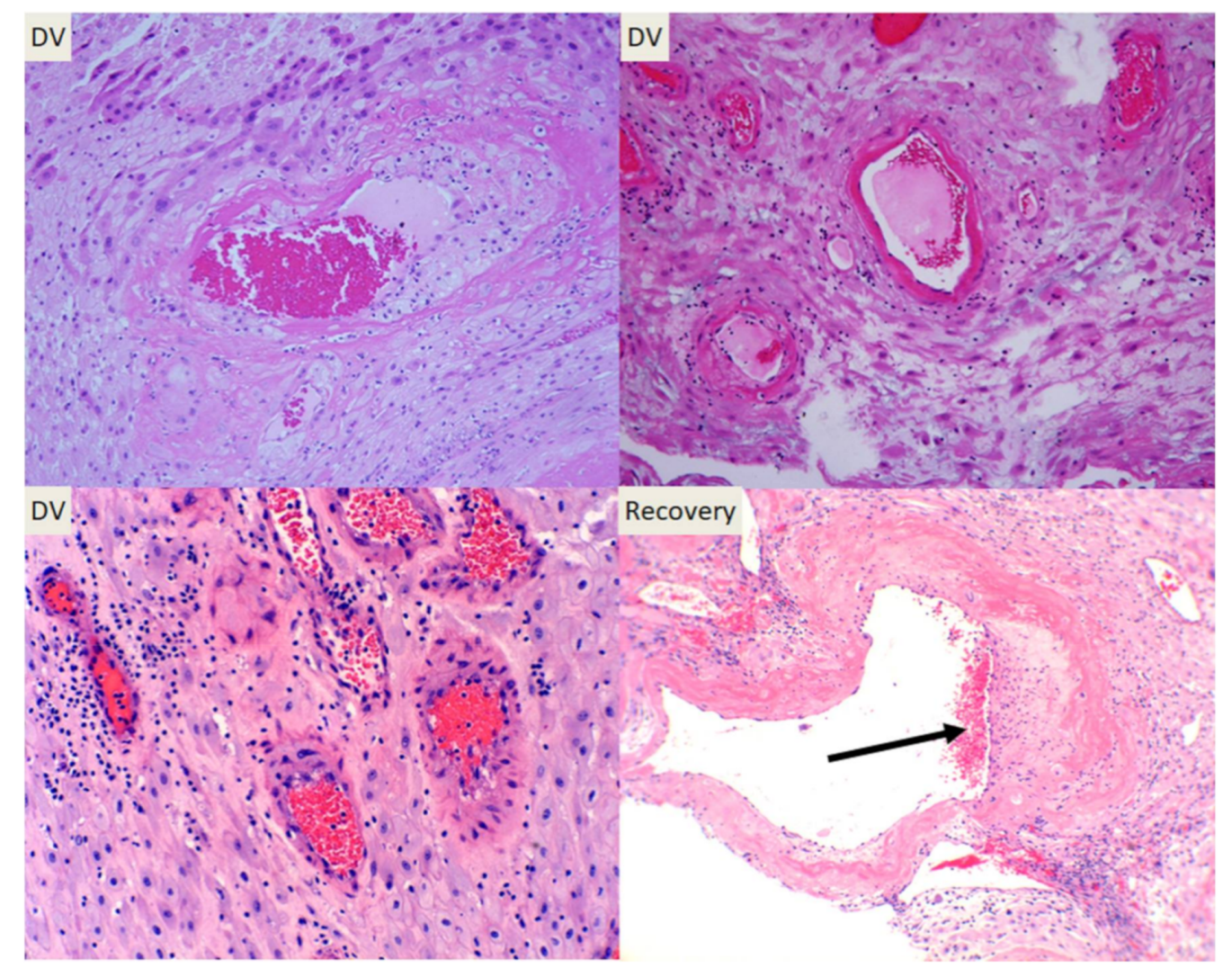

Figure 2. Classic decidual vasculopathy including atherosis, fibrinoid medial necrosis and mural arterial hypertrophy/hyperplasia can be identified either at the central portion or peripheral portion of maternal surface. Regenerating smooth muscle cells and endothelium presented (black arrow) as "cushion". DV, decidual vasculopathy.

The endovascular and intramural trophoblasts associated with decidual vasculopathy can be identified by using immunostaining of CD56 (Figure 3). The endothelial and smooth muscle cell regeneration/restoration within the lumen of spiral artery were detected by using WT1 and smooth muscle myosin heavy chain (SMYOHC). Co-existence of endovascular trophoblasts and endothelial cells was seen occasionally within the same spiral artery. Intramural trophoblasts with overlying endothelial cells were more frequently identified (Figure 3).

There were 72 placentas with decidual vasculopathy in the central region, including 59 cases with classic type decidual vasculopathy (atherosis and fibrinoid medial necrosis) and 13 cases with mixed type decidual vasculopathy (classic type and arterial mural hypertrophy; Table 2). In total, 88 placentas in the peripheral regions showed the presence of decidual vasculopathy, including 73 placentas with classic type and 15 placentas with mixed type vasculopathy (Table 2). 
Table 2. Decidual vasculopathy type and location with frequency of recovery.

\begin{tabular}{cccccccc}
\hline Decidual Vasculopathy & Central & Peripheral & Both $\mathbf{( P )}$ & Recovery (C) & Recovery (P) & Both (R) & $\boldsymbol{p}$-Value \\
\hline Total & 72 & 88 & 56 & 51 & 23 & 13 & $<0.0001$ \\
Classic & 59 & 73 & 45 & 41 & 19 & 11 & $<0.0001$ \\
Mixed & 13 & 15 & 11 & 10 & 4 & 2 & $=0.118$ \\
\hline
\end{tabular}

Table 2: Frequency of decidual vasculopathy and vascular recovery (regeneration/restoration) in the central and the peripheral regions of the placentas with Fisher exact probability test. Both $(\mathrm{P})$ depicts the presence of decidual vasculopathy at both central and peripheral regions. Both (R) depicts the presence of regeneration/restoration in both central and peripheral regions.

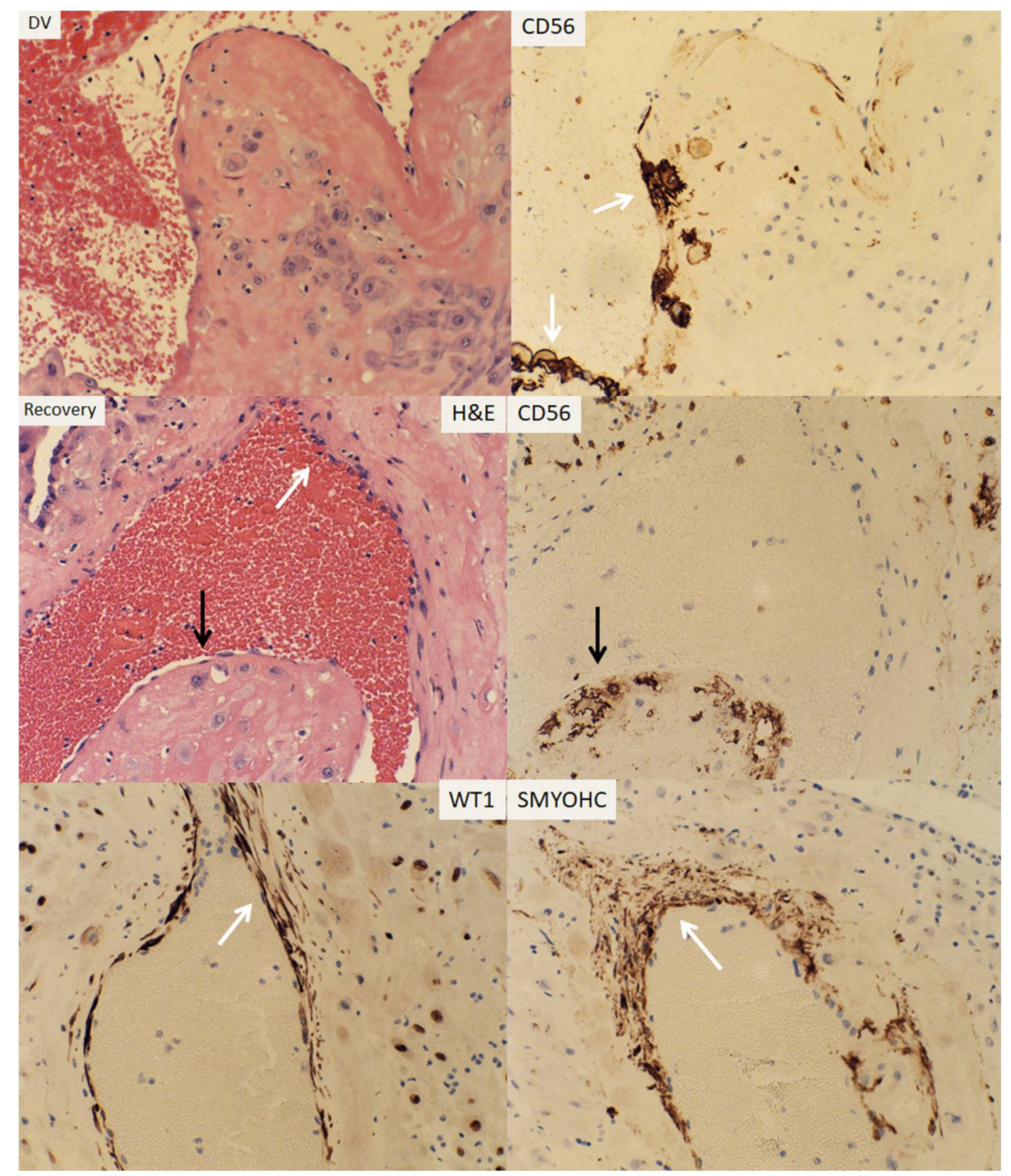

Figure 3. Representative sections of decidual vasculopathy and vascular regeneration/restoration with corresponding immunostaining patterns. Partially restored vessels contained endovascular and intramural trophoblasts as well as endothelial cells (top and middle panels). Fully restored vessels contained only endothelial cells and smooth muscle cells with no endovascular or intramural trophoblasts. The white arrows point to regenerating endothelial cells and smooth muscle cells and black arrows point to residual endovascular/intramural trophoblasts. All section were at 200× magnification. SMYOHC, smooth muscle myosin heavy chain.

Frequently both the central and peripheral regions of the placentas showed the presence of decidual vasculopathy but the vascular recovery (regeneration/restoration) was identified at a much lower frequency at the peripheral region than in the central region (Table 1; Table 2; $p<0.0001$, Fisher exact probability test, two tailed). 
We examined one hysterectomy specimen containing the placenta at the 39 weeks with placenta increta within the previous C-section scar for vascular changes within the decidua and superficial myometrium (Figure 4). The patient didn't have clinical history of preeclampsia or chronic hypertension. All of the sections of the myometrium and placental tissue were from the central areas. Decidual vasculopathy can be easily identified with various degrees of vascular regeneration/restoration within the decidual tissue (Figure 4).

The residual endovascular and intramural trophoblasts were detected by immunostaining for CD56, and the regenerating endothelial cells and smooth muscle cells by WT1 and SMYOHC (Figure 4). In contrast, the vascular changes within the superficial myometrium deep to the decidual tissue showed only regenerating arterial wall with endothelium and smooth muscle cells. No CD56-positive trophoblasts were detected in the myometrium section (Figure 5).

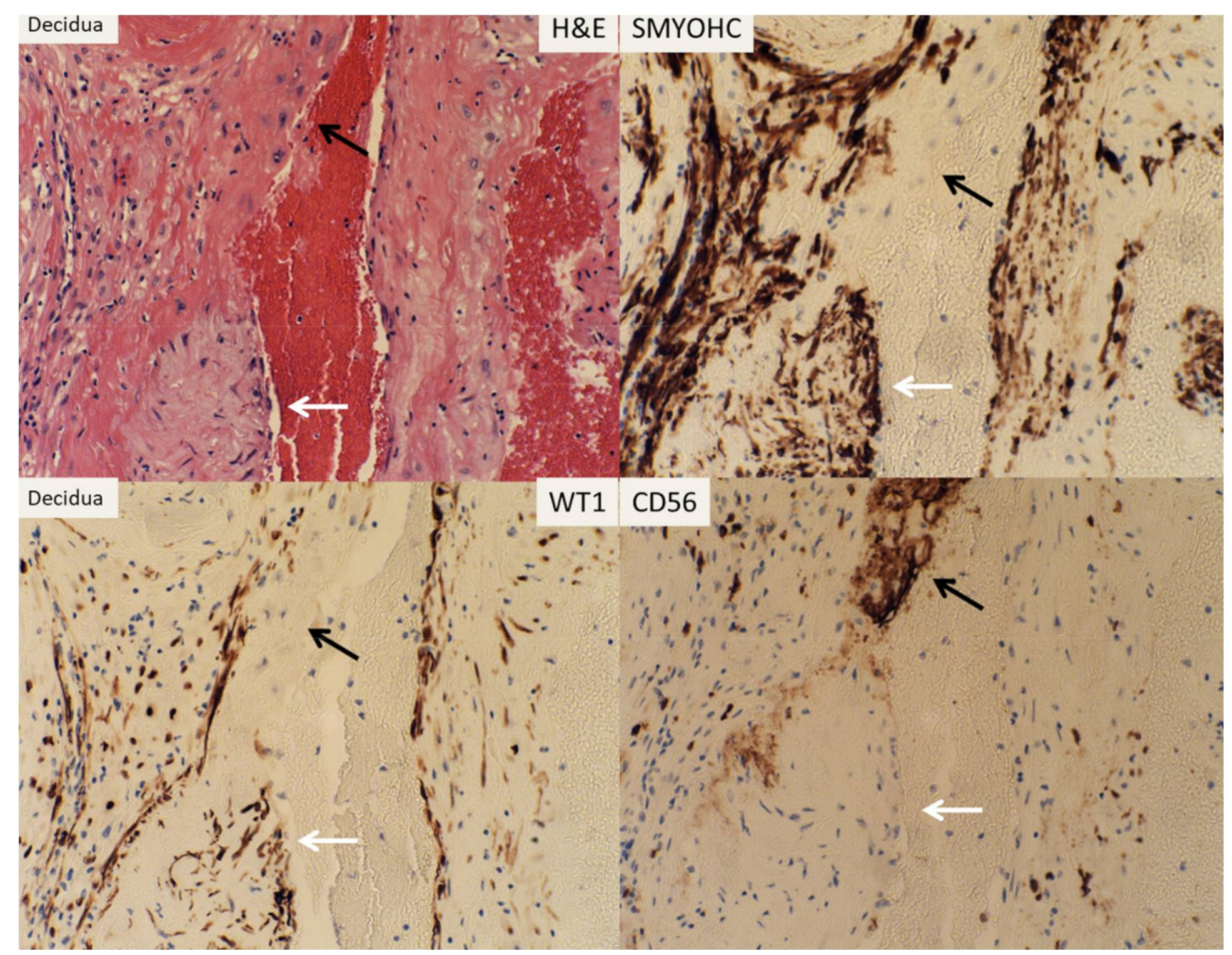

Figure 4. Histologic examination with immunostaining of vascular changes of spiral artery from hysterectomy specimen containing decidua. The white arrows point to regenerating endothelial cells and smooth muscle cells and the black arrows point to residual endovascular/intramural trophoblasts. SMYOHC, smooth muscle myosin heavy chain. All sections were at 200× magnification. 


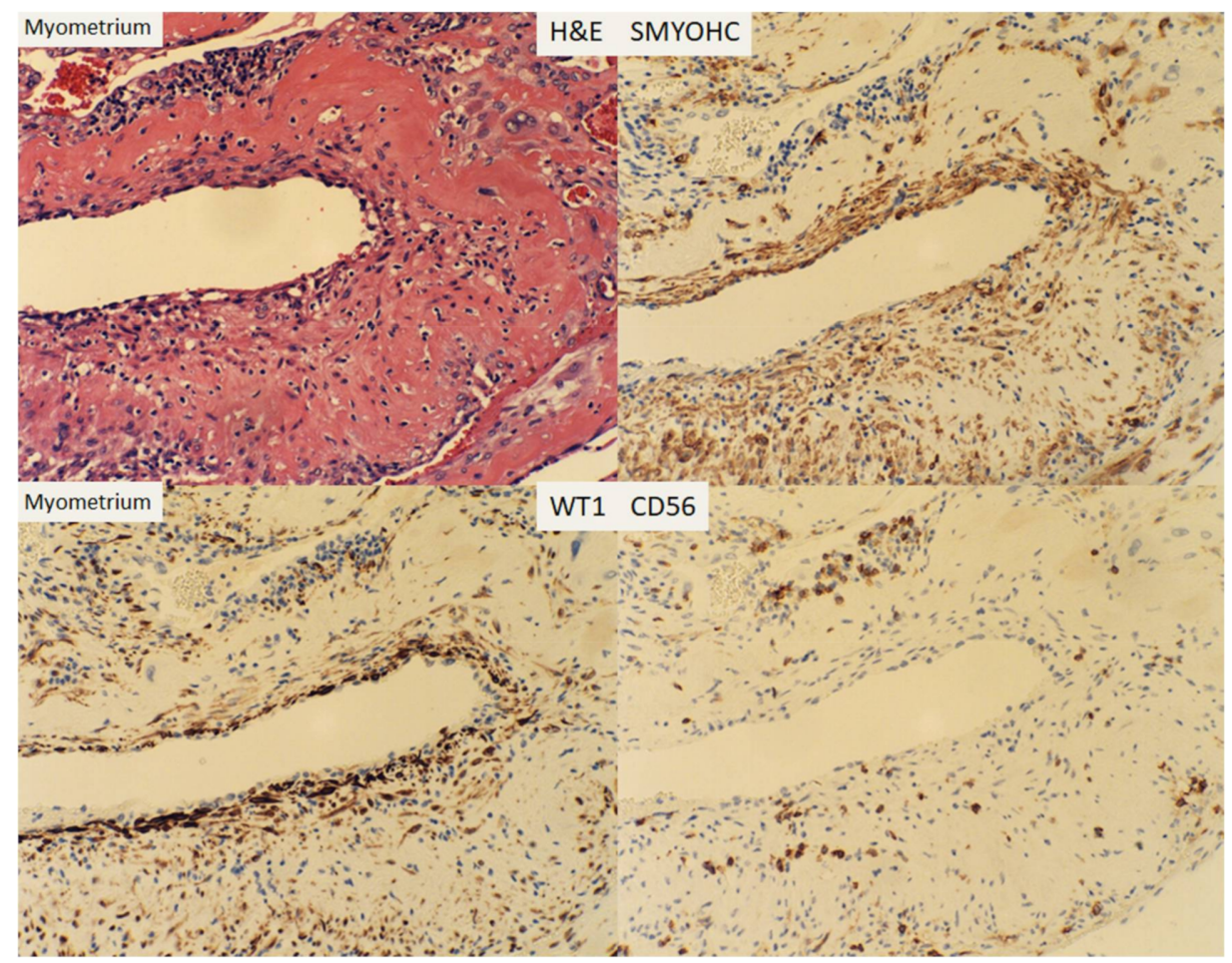

Figure 5. Histologic examination with immunostaining of vascular changes of spiral artery from hysterectomy specimen containing superficial myometrium. The white arrows point to regenerating endothelial cells and smooth muscle cells and the black arrows point to residual endovascular/intramural trophoblasts. SMYOHC, smooth muscle myosin heavy chain. All sections were at $200 \times$ magnification.

\section{Discussion}

In current study we attempted to evaluate the maternal surface for decidual vasculopathy in a spatial fashion to answer the question of whether there is a difference in morphologic changes of spiral artery that reflects the temporal vascular transformation. The study is based on the hypothesis that the central portion of the placenta is formed first with lateral expansion for continuous placental growth to term. There are few studies devoted to the lateral growth of placenta and spiral artery remodeling in a spatiotemporal fashion although much of the effort is focused on the trophoblasts dependent remodeling of spiral artery in temporal fashion [11,12]. Placenta is a transient organ, and maternal vascular transformation during pregnancy undergoes significant variations from the early "trophoblastic plugging", disappearance of smooth muscle wall and fibrinoid medial necrosis to the disappearance of endovascular trophoblasts, and regeneration/restoration of endothelium and smooth muscle wall after delivery. Our previous study demonstrated that the intramural and endovascular trophoblasts persisted from early implantation to late pregnancy with formation of morphologic features of classic decidual vasculopathy including acute atherosis and medial fibrinoid necrosis $[18,22]$. The link between decidual vasculopathy at late gestation to spiral artery remodeling at implantation raises the possibility of "failure to die" of endovascular trophoblasts as a mechanism of pathogenesis of decidual vasculopathy. It also raises the questions of whether the death of endovascular trophoblasts is synchronously regulated through either maternal circulating factors (systemic) or specific interaction with the endothelial cells of the proximal artery (local) or both [22,23]. It is frequently observed that the endovascular trophoblasts and the endothelium are mutually exclusive within the arterial lumen, but the intramural trophoblasts and endothelium can co-exist with overlying endothelium on the surface and underlying intramural trophoblasts within the arterial wall. This observation suggests that the endothelium regenerates/restores first after spiral artery remodeling of arterial wall. It is also consistent with the previous study that the endothelial progenitor cells give rise to the smooth muscle wall of the coronary artery $[24,25]$. 
The formation of placental cotyledons from the center to the periphery is gradual and time-dependent. However, it is unclear if the formation of cotyledons is continuous, spanning the entire gestational age, although the placental weight increase occurs most in the third trimester. The connection of the cotyledons to maternal circulation through spiral artery remodeling from the center to the periphery should also theoretically be time-dependent. Our current data showed there is a statistically significant difference regarding the frequency of decidual vasculopathy between the central portion and peripheral portion of the placenta, as well as the regenerating/restorative features of the spiral artery after decidual vasculopathy. The dynamic changes of decidual vascular transformation in a spatiotemporal fashion help the interpretation of decidual vasculopathy during placental examination after delivery, as the placental examination only captures a snapshot of vascular changes of the spiral artery in specific time.

Pathogenesis of decidual vasculopathy is controversial and the factors affecting the cell death program of endovascular trophoblasts and relationship to regeneration/restoration of endothelium and smooth muscle wall are unclear at this time. Wilms tumor (WT1) gene and Wilms tumor associated protein (WTAP) gene expressions have been shown to be important for vascular and smooth muscle regeneration $[21,26,27]$. Smooth muscle regeneration in coronary arteries is dependent upon WT1 gene expression from the endothelial progenitor cells [24]. WT1 gene expression has been studied extensively in other cellular and animal models, and WT1 gene expression in endometrium during the menstrual cycle and gestation was also attempted [20]. It is highly likely that quantitative measures are needed for WT1 gene expression in gestational vascular transformation to determine the roles of WT1 and WTAP genes in regeneration of endothelium and smooth muscles of the spiral artery locally, as a basal level of WT1 expression was detected within the endometrial stromal cells throughout the menstrual cycles and pregnancy [20]. It is also likely that unknown maternal circulating factors will influence the WT1 gene expression globally in a relatively synchronous manner. To search factors affecting WT1 gene expression within the regenerating endothelium and smooth muscles of spiral artery, using in vitro models will likely provide useful information in pathogenesis of decidual vasculopathy and preeclampsia.

The controversy of vascular changes within the decidua and the superficial myometrium remains as placental bed biopsy predominantly studied the vessels within the myometrium $[15,28]$. Myometrial vessels continue distally to become the decidual spiral artery. Vascular transformation during early pregnancy starts at the decidual portion and progresses proximally to the myometrial portion. Consequently, regeneration during late pregnancy or after delivery starts proximally. Our current study only captured the decidual vascular changes at late gestation, and spatiotemporal vascular transformation at early stages of pregnancy requires more research. The past research effort has been focused on the decidual arteries and the involvement of decidual veins by the trophoblastic dependent and trophoblastic independent remodeling mechanisms have not been studied extensively, as remodeling of decidual veins has a clearly different implication to the function of placental development.

\section{Conclusions}

There is a statistically significant difference in both the incidence of decidual vasculopathy between the central and peripheral placenta and the regeneration/restoration of endothelium and smooth muscle of spiral artery due to the lateral growth pattern and corresponding spiral artery remodeling. Dynamic and spatiotemporal view of the spiral artery remodeling during pregnancy provides useful information in development of decidual vasculopathy at late gestation.

Funding: This research received no external funding.

Acknowledgments: The author is thankful for Nina Yermolayeva, Cheryl Hernandez, Elizabeth Feygina, and Missver Jocson for their technical assistance in preparation of placental specimens.

Conflicts of Interest: The author declares no conflict of interest. 


\section{References}

1. Benirschke, K.; Burton, G.J.; Baergen, R.N. Pathology of the Human Placenta, 6th ed.; Springer: New York, NY, USA, 2012.

2. Hutcheon, J.A.; McNamara, H.; Platt, R.W.; Benjamin, A.; Kramer, M.S. Placental weight for gestational age and adverse perinatal outcomes. Obstet. Gynecol. 2012, 119, 1251-1258. [CrossRef] [PubMed]

3. Salafia, C.M.; Charles, A.K.; Maas, E.M. Placenta and fetal growth restriction. Clin. Obstet. Gynecol. 2006, 49, 236-256. [CrossRef] [PubMed]

4. Salafia, C.M.; Maas, E.; Thorp, J.M.; Eucker, B.; Pezzullo, J.C.; Savitz, D.A. Measures of placental growth in relation to birth weight and gestational age. Am. J. Epidemiol. 2005, 162, 991-998. [CrossRef] [PubMed]

5. Salafia, C.M.; Pezzullo, J.C.; Charles, A.K.; Ernst, L.M.; Maas, E.M.; Gross, B.; Pijnenborg, R. Morphometry of the basal plate superficial uteroplacental vasculature in normal midtrimester and at term. Pediatr. Dev. Pathol. 2005, 8, 639-646. [CrossRef]

6. Bonds, D.R.; Gabbe, S.G.; Kumar, S.; Taylor, T. Fetal weight/placental weight ratio and perinatal outcome. Am. J. Obstet. Gynecol. 1984, 149, 195-200. [CrossRef]

7. Bonds, D.R.; Mwape, B.; Kumar, S.; Gabbe, S.G. Human fetal weight and placental weight growth curves. A mathematical analysis from a population at sea level. Biol. Neonate 1984, 45, 261-274. [CrossRef]

8. Raff, M.C.; Durand, B.; Gao, F.B. Cell number control and timing in animal development: The oligodendrocyte cell lineage. Int. J. Dev. Biol. 1998, 42, 263-267.

9. Conlon, I.J.; Dunn, G.A.; Mudge, A.W.; Raff, M.C. Extracellular control of cell size. Nat. Cell Biol. 2001, 3, 918-921. [CrossRef]

10. Raff, M.C. Size control: The regulation of cell numbers in animal development. Cell 1996, 86, $173-175$. [CrossRef]

11. Craven, C.M.; Zhao, L.; Ward, K. Lateral placental growth occurs by trophoblast cell invasion of decidual veins. Placenta 2000, 21, 160-169. [CrossRef]

12. Pijnenborg, R.; Dixon, G.; Robertson, W.B.; Brosens, I. Trophoblastic invasion of human decidua from 8 to 18 weeks of pregnancy. Placenta 1980, 1, 3-19. [CrossRef]

13. Pijnenborg, R.; Vercruysse, L.; Hanssens, M. The uterine spiral arteries in human pregnancy: Facts and controversies. Placenta 2006, 27, 939-958. [CrossRef] [PubMed]

14. Cartwright, J.E.; Fraser, R.; Leslie, K.; Wallace, A.E.; James, J.L. Remodelling at the maternal-fetal interface: Relevance to human pregnancy disorders. Reproduction 2010, 140, 803-813. [CrossRef] [PubMed]

15. Robertson, W.B.; Khong, T.Y.; Brosens, I.; De Wolf, F.; Sheppard, B.L.; Bonnar, J. The placental bed biopsy: Review from three European centers. Am. J. Obstet. Gynecol. 1986, 155, 401-412. [CrossRef]

16. Khong, T.Y.; Chambers, H.M. Alternative method of sampling placentas for the assessment of uteroplacental vasculature. J. Clin. Pathol. 1992, 45, 925-927. [CrossRef] [PubMed]

17. Khong, T.Y.; Mooney, E.E.; Ariel, I.; Balmus, N.C.; Boyd, T.K.; Brundler, M.A.; Derricott, H.; Evans, M.J.; Faye-Petersen, O.M.; Gillan, J.E.; et al. Sampling and Definitions of Placental Lesions: Amsterdam Placental Workshop Group Consensus Statement. Arch. Pathol. Lab. Med. 2016, 140, 698-713. [CrossRef] [PubMed]

18. Zhang, P. Phenotypic Switch of Endovascular Trophoblasts in Decidual Vasculopathy with Implication for Preeclampsia and Other Pregnancy Complications. Fetal. Pediatr. Pathol. 2020, 1-20. [CrossRef] [PubMed]

19. Hertig, A. Vascular pathology in the hypertensive albuminuric toxemias of pregnancy. Clinics 1945, 4, 602-614.

20. Zhang, P. Expression of Wilm's Tumor Gene in Endometrium with Potential Link to Gestational Vascular Transformation. Reprod. Med. 2020, 1, 17-31. [CrossRef]

21. Hastie, N.D. Wilms' tumour 1 (WT1) in development, homeostasis and disease. Development 2017, 144, 2862-2872. [CrossRef]

22. Zhang, P. Decidual Vasculopathy in Preeclampsia and Spiral Artery Remodeling Revisited: Shallow Invasion versus Failure of Involution. AJP Rep. 2018, 8, e241-e246. [CrossRef] [PubMed]

23. Zhang, P. Decidual vasculopathy and spiral artery remodeling revisited II: Relations to trophoblastic dependent and independent vascular transformation. J. Matern. Fetal Neonatal Med. 2020, 1-7. [CrossRef]

24. Martínez-Estrada, O.M.; Lettice, L.A.; Essafi, A.; Guadix, J.A.; Slight, J.; Velecela, V.; Hall, E.; Reichmann, J.; Devenney, P.S.; Hohenstein, P.; et al. Wt1 is required for cardiovascular progenitor cell formation through transcriptional control of Snail and E-cadherin. Nat. Genet. 2010, 42, 89-93. [CrossRef] [PubMed] 
25. Chau, Y.Y.; Hastie, N. Wt1, the mesothelium and the origins and heterogeneity of visceral fat progenitors. Adipocyte 2015, 4, 217-221. [CrossRef]

26. Small, T.W.; Penalva, L.O.; Pickering, J.G. Vascular biology and the sex of flies: Regulation of vascular smooth muscle cell proliferation by wilms' tumor 1-associating protein. Trends Cardiovasc. Med. 2007, 17, 230-234. [CrossRef] [PubMed]

27. Small, T.W.; Bolender, Z.; Bueno, C.; O’Neil, C.; Nong, Z.; Rushlow, W.; Rajakumar, N.; Kandel, C.; Strong, J.; Madrenas, J.; et al. Wilms' tumor 1-associating protein regulates the proliferation of vascular smooth muscle cells. Circ. Res. 2006, 99, 1338-1346. [CrossRef] [PubMed]

28. Brosens, I.; Puttemans, P.; Benagiano, G. Placental bed research: I. The placental bed: From spiral arteries remodeling to the great obstetrical syndromes. Am. J. Obstet. Gynecol. 2019, 221, 437-456. [CrossRef]

Publisher's Note: MDPI stays neutral with regard to jurisdictional claims in published maps and institutional affiliations.

(C) 2020 by the author. Licensee MDPI, Basel, Switzerland. This article is an open access article distributed under the terms and conditions of the Creative Commons Attribution (CC BY) license (http://creativecommons.org/licenses/by/4.0/). 